\title{
Erratum to: Elastic Waves in Swelling Porous Media
}

\author{
S. K. Tomar · Suraj Goyal
}

Published online: 5 April 2014

(C) Springer Science+Business Media Dordrecht 2014

\section{Erratum to: Transp Porous Med (2013) 100:39-68 DOI 10.1007/s11242-013-0204-4}

In our recent paper, cited above, the Sect. 4.4 contains the formulae of $E_{i}$ and $E_{i j}$ given by Eqs. (61) and (63) respectively. These formulae should be preceded by a negative sign, that is,

$$
\begin{gathered}
E_{i}=-\frac{\left\langle P_{i}^{e}\right\rangle}{\left\langle P_{0}^{e}\right\rangle}, \\
E_{i j}=-\frac{\left\langle P_{i j}^{e}\right\rangle}{\left\langle P_{00}^{e}\right\rangle}=-\frac{\Re\left(F_{i j} R_{i} R_{j}^{*}\right)}{\Re\left(F_{00}\right)},
\end{gathered}
$$

where $(i, j=0,1, \ldots, 5)$. Negative sign is taken because the direction of the reflected waves is opposite to that of incident wave. The Eq. (65) describing the energy conservation law, now takes the form

$$
E_{I R}+E_{R R}+\sum_{r=1}^{5} E_{r r}=-E_{00}=1 .
$$

Also, under Appendix 3, the expression of elements $F_{i 0}$ of matrix $F$ contains a typographical error. There should be $d_{\alpha 1}^{*}$ instead of $k^{*}$ in the first part. The correct expression of $F_{i 0}$ is given by

$$
F_{i 0}=D_{1}\left[\left(L_{i}+f_{1}^{*} M_{i}+g_{1}^{*} N_{i}\right) k_{\alpha i}^{2} d_{\alpha 1}^{*}-2 k\left(\bar{a}_{11}+f_{i} f_{1}^{*} \bar{a}_{22}\right)\left(k d_{\alpha 1}^{*}+k^{*} d_{\alpha i}\right)\right] .
$$

The online version of the original article can be found under doi:10.1007/s11242-013-0204-4.

S. K. Tomar $(\bowtie) \cdot$ S. Goyal

Department of Mathematics, Panjab University, Chandigarh 160 014, India e-mail: sktomar66@gmail.com

S. Goyal

e-mail: surajgoya187@gmail.com 
(a)

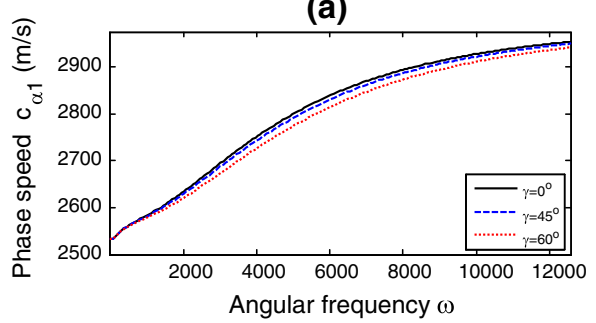

(c)

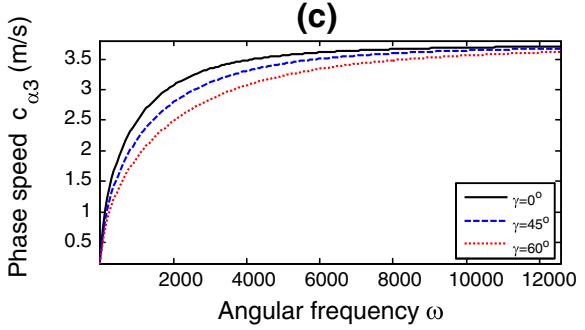

(e)

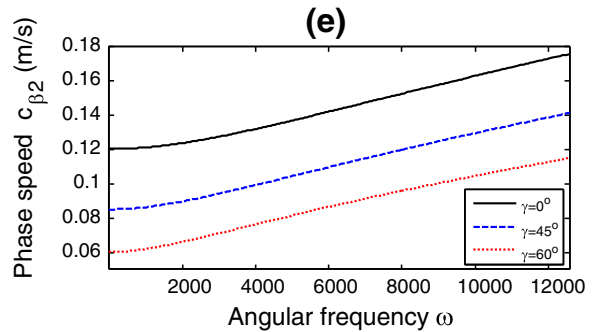

(b)

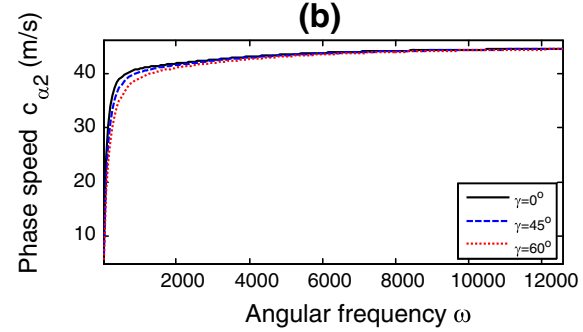

(d)

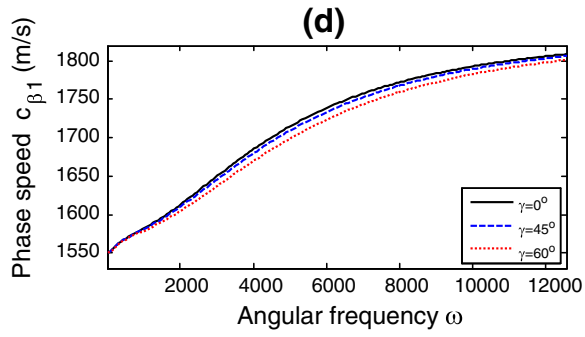

Fig. 2 Plots of phase speeds of dilatational and shear waves as a function of angular frequency

Moreover, while re-visiting the program of numerical computations meant for the purpose, we have noticed an error in MATLAB coding. After correcting the error, we found that there are significant changes in the magnitudes and trends of the graphs. The numerical computations are done afresh and the revised graphs are presented here through Figs. 2, 3, 4, 5, 6, 7, 8, 9, 10 , and 11 .

Figure $2 \mathrm{a}-\mathrm{e}$ shows the variation of phase speeds of all the dilatational and shear waves, while variation of their corresponding attenuations with angular frequency varying from $2 \pi \times 1 \mathrm{~Hz}$ to $2 \pi \times 2 \mathrm{kHz}$ are exhibited through Fig. $3 \mathrm{a}-\mathrm{e}$ at three fixed values of attenuation angle, namely, $0^{\circ}, 45^{\circ}$ and $60^{\circ}$. In these figures, the solid curve corresponds to a homogeneous wave. In these corrected graphs, we see that the magnitude of phase speeds corresponding to the fast moving $P_{1}$ and $S_{1}$-waves and their trends with angular frequency (or frequency) are affected to a great extent. Both of these waves behave alike with frequency, i.e., their phase speeds increase gradually with increase in the considered frequency range. At higher frequency range (beyond $20 \mathrm{kHz}$ ), the phase speeds of $P_{1}$ and $S_{1}$ waves become almost constant with values $c_{\alpha 1} \sim 3.0088 \times 10^{3} \mathrm{~m} / \mathrm{s}$ and $c_{\beta 1} \sim 1.8425 \times 10^{3} \mathrm{~m} / \mathrm{s}$ respectively. And the effect of inhomogeneity (angle of attenuation) on the phase speeds is almost nil in this range. The behavior of phase speeds of other three waves (i.e., $P_{2}, P_{3}$ and $S_{2}$ ) with frequency parameter remains almost same as was depicted in the above cited paper except a little change in their magnitudes. Again, we see that the phase speed of $S_{2}$ wave is not only 
(a)

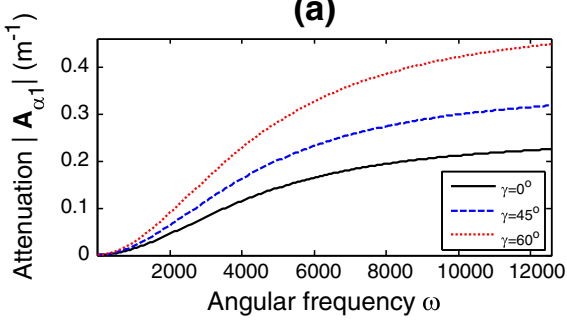

(c)

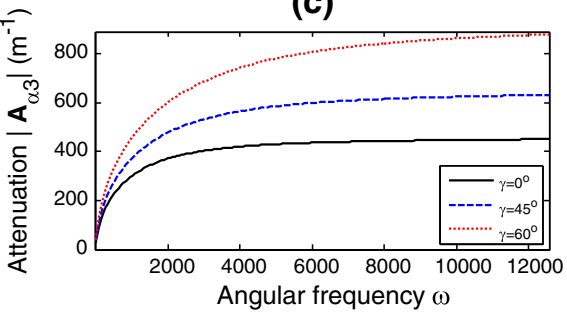

(e)

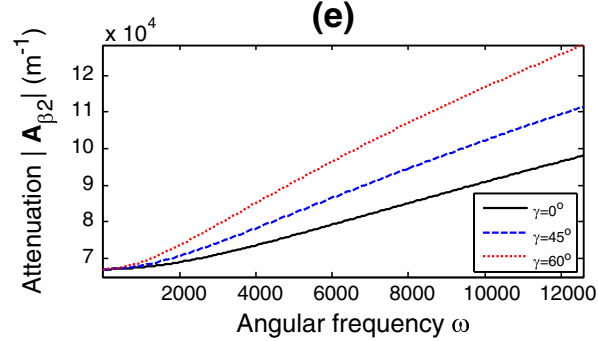

(b)

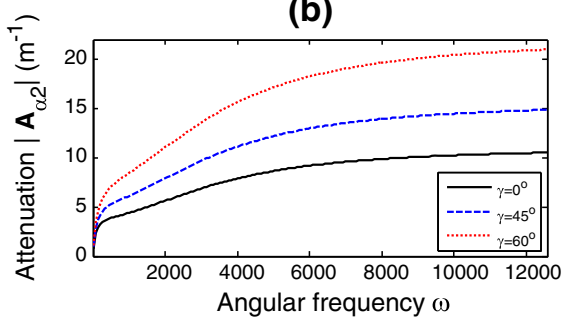

(d)

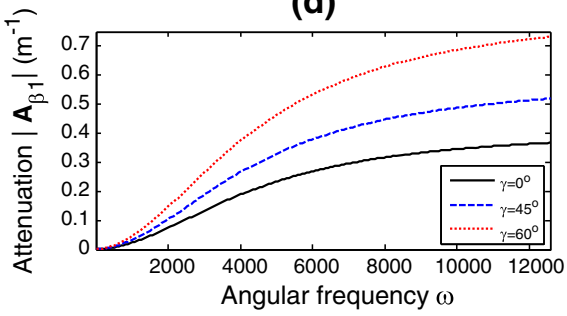

Fig. 3 Plots of attenuation coefficients of dilatational and shear waves as a function of angular frequency

poorly dependent on frequency, but its value is also very small in comparison to those of the other waves. From Fig. 3, it is clear that the fast moving $P_{1}$ and $S_{1}$ waves are least attenuated and their behavior with frequency is almost similar, while the slowest $S_{2}$ wave is highly attenuated. It is also interesting to note that for each of the five propagating waves, an increase of frequency and inhomogeneity (angle of attenuation) increases its attenuation. However, the effect of inhomogeneity is more prominent at higher frequency range. A comparison of Figs. 2 and 3 shows that an increase in inhomogeneity at a given frequency results a decrease in the phase speed of the propagating wave but an increase in its corresponding attenuation. The phase speeds (attenuations) of inhomogeneous waves are always less (greater) than those of the corresponding homogeneous waves. These results are in concurrence with those given in Borcherdt (2009, pp. 37). The second shear $S_{2}$-wave is seen to propagate very slow, but highly attenuated as compared to other waves, that is, it dies out quickly and may not be easy to capture.

Figure $4 \mathrm{a}-\mathrm{e}$ depicts the variation of the absolute values of various reflection coefficients with the angle of incidence of $P_{1}$-wave at three fixed values of frequency parameter, namely, $100 \mathrm{~Hz}, 200 \mathrm{~Hz}$ and $300 \mathrm{~Hz}$ at $45^{\circ}$ angle of attenuation, in case of open-pore boundary, while Fig. $4 \mathrm{f}-\mathrm{j}$ depicts the same in case of sealed-pore boundary. From the plots of both the boundary surface cases, it is crystal clear that except $P_{1}$ and $S V_{1}$ waves, the existence of the other reflected dilatational and shear waves (i.e. $P_{2}, P_{3}$ and $S V_{2}$ ) is namesake. In particular 
(a)

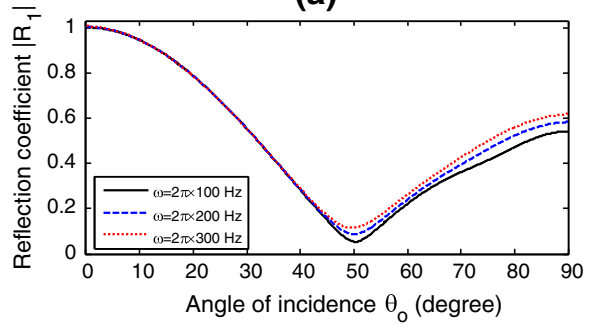

(b)

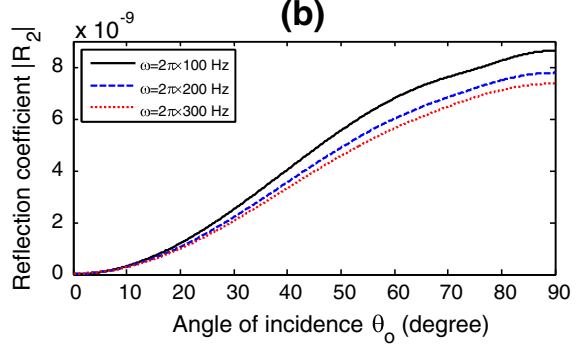

(c)

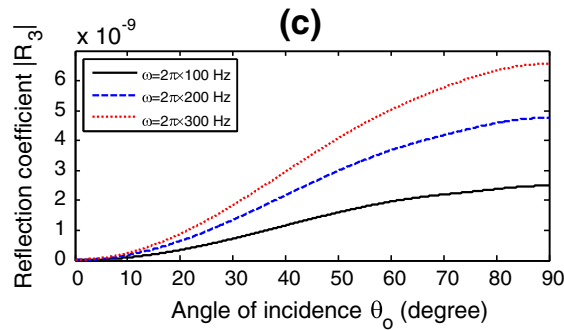

(d)

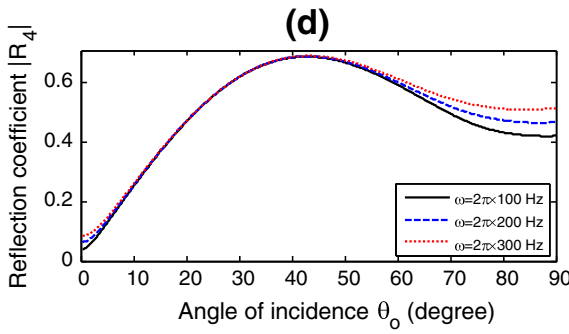

(e)

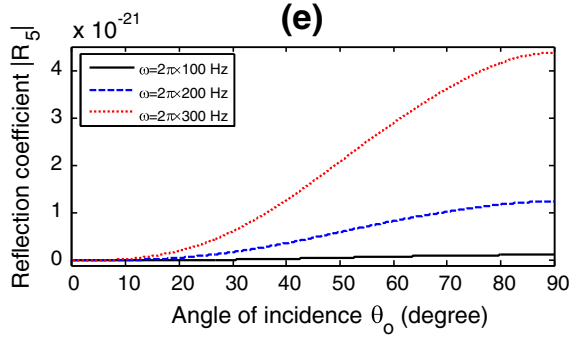

(f)

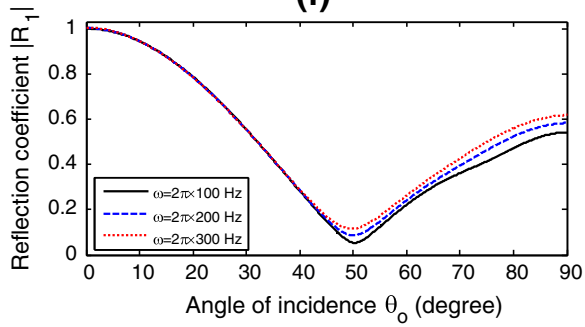

(g)

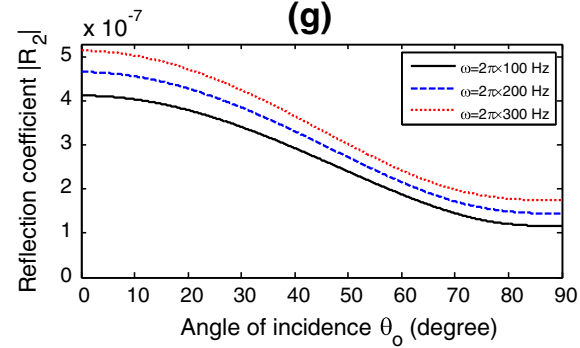

(h)

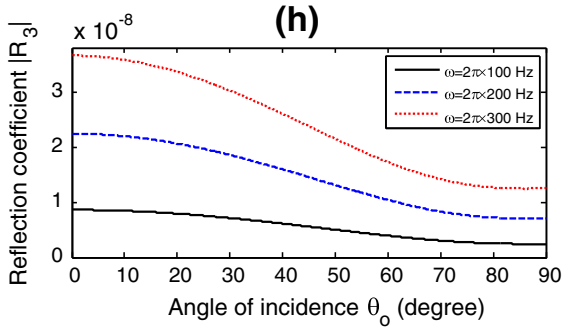

(i)

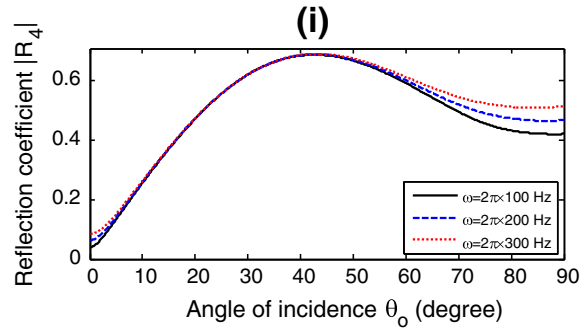

(j)

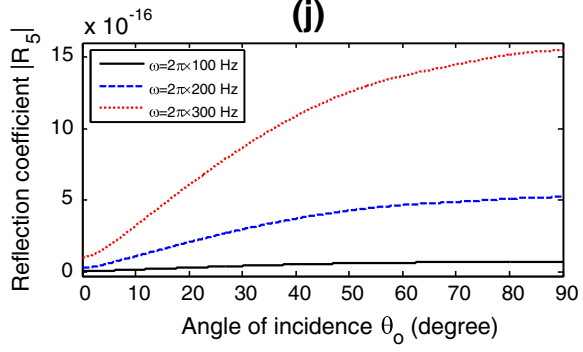

Fig. 4 Reflection coefficients: a-e open-pore boundary, $\mathbf{f}-\mathbf{j}$ sealed-pore boundary $\left(\right.$ at $\left.\gamma_{0}=45^{\circ}\right)$ 
(a)

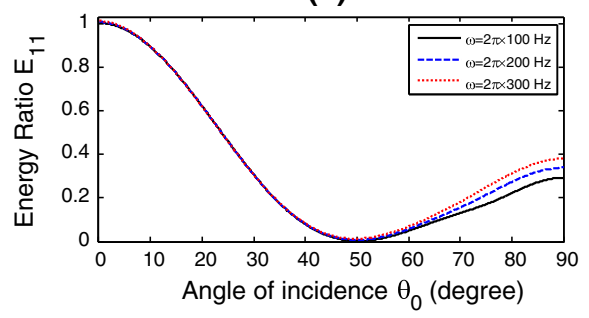

(c)

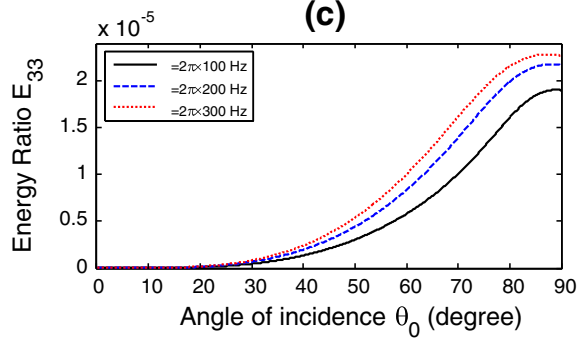

(e)

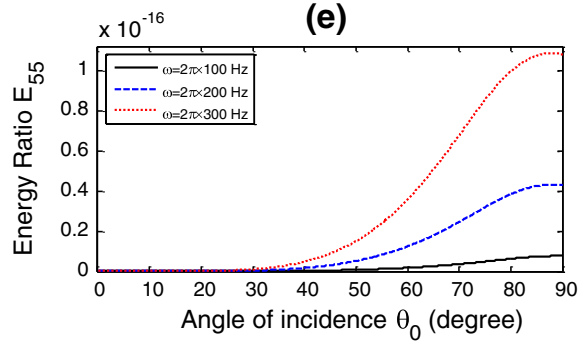

(g)

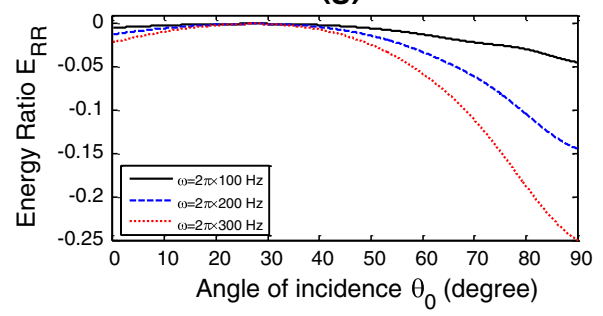

(b)

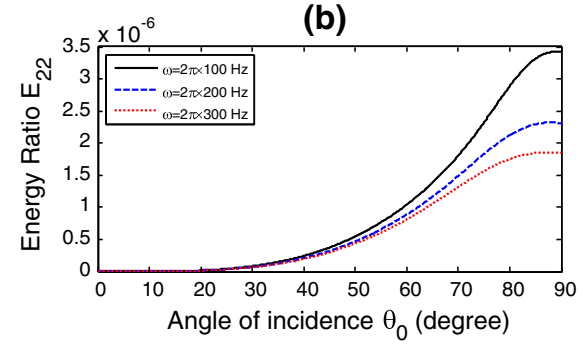

(d)

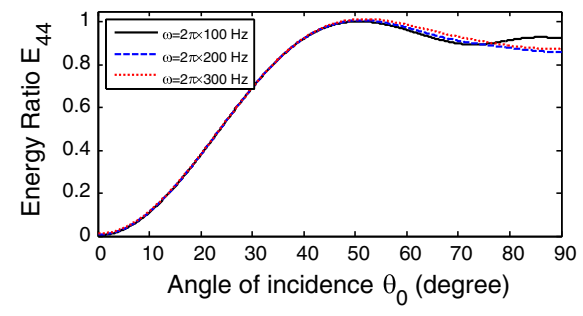

(f)

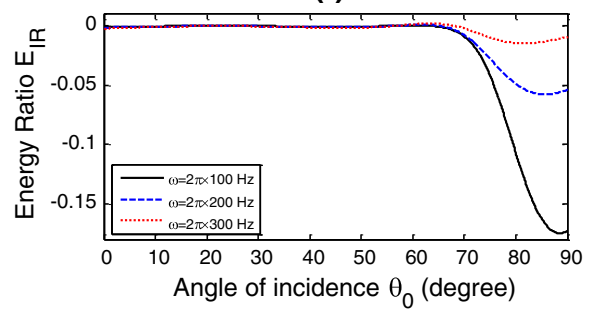

Fig. 5 Energy ratios with angle of incidence for open-pore boundary case (at $\gamma_{0}=45^{\circ}$ )

the coefficient $\left|R_{5}\right|$ is very very small. This may be due to very small value of the coefficient $\mu_{v}$ chosen in the model and the existence of the second shear wave heavily depends on the quantity $\mu_{\nu}$. It is also clear that as the frequency of incident wave increases, the reflection coefficients also increase, in general. In both open and sealed boundary surfaces, the reflection coefficient $\left|R_{1}\right|$ corresponding to reflected $P_{1}$ wave attains its minimum value $(\sim 0.0543)$ as $\theta_{0}$ approaches to $50^{\circ}$, while the coefficient $\left|R_{4}\right|$ corresponding to reflected $S V_{1}$ wave attains its maximum value $(\sim 0.6888)$ as $\theta_{0}$ approaches to $43^{\circ}$, for an incident wave having $100 \mathrm{~Hz}$ frequency. At normal incidence, only the reflection coefficient corresponding to $P_{1}$ wave is found to exist significantly. It is also observed that the reflection coefficients corresponding 
(a)

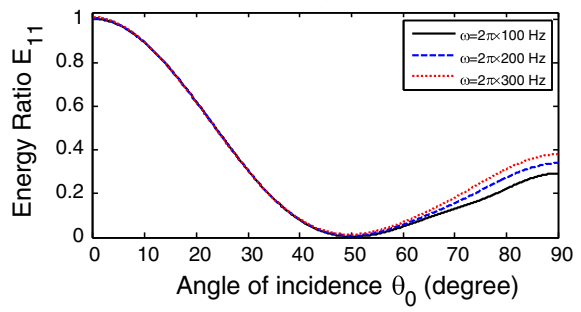

(c)

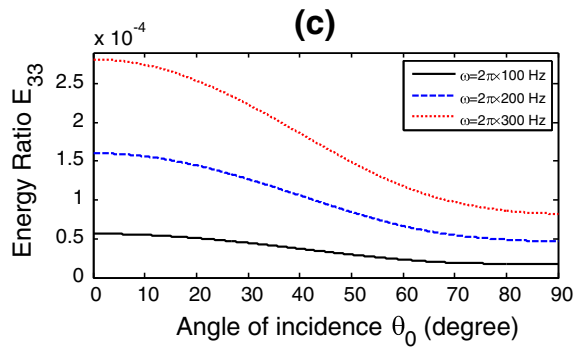

(e)

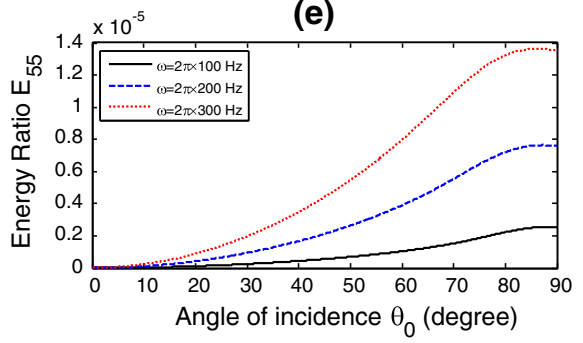

(g)

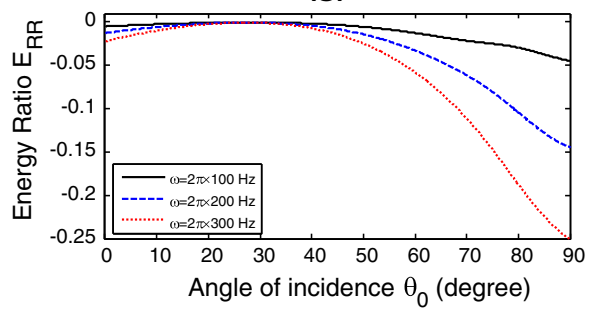

(b)

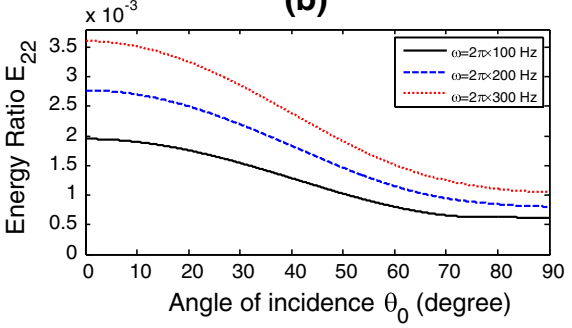

(d)

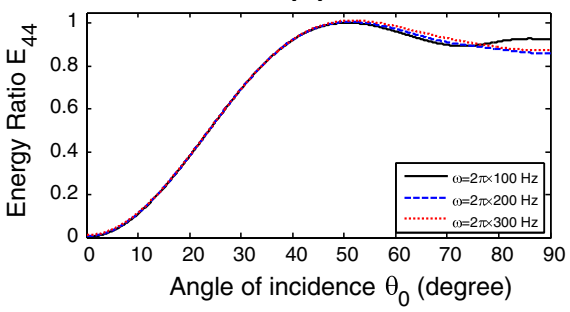

(f)

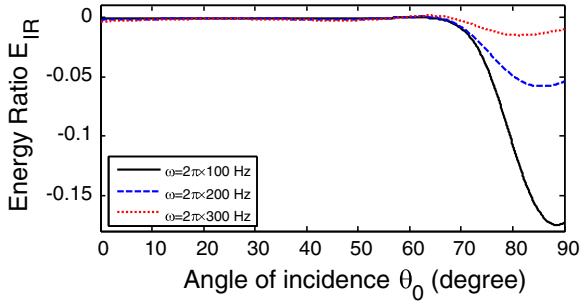

Fig. 6 Energy ratios with angle of incidence for sealed-pore boundary case (at $\gamma_{0}=45^{\circ}$ )

to $P_{1}$ and $S V_{1}$ waves remain same in both cases of boundary surface, while the reflection coefficients corresponding to other reflected waves are affected and these coefficients become more significant in case of sealed pore boundary surface. The dependence of $\left|R_{2}\right|$ and $\left|R_{3}\right|$ on the angle of incidence is found to be reverse in the two boundary surface cases.

Figure 5a-e depicts the variation of energy ratios corresponding to various reflected waves at three fixed values of frequency parameter $(100 \mathrm{~Hz}, 200 \mathrm{~Hz}, 300 \mathrm{~Hz})$ at $45^{\circ}$ angle of attenuation, in case of open-pore boundary, while Fig. 6a-e depicts the same in case of sealed-pore boundary. Figure 5f,g depicts the interacting energies in open-pore case and Fig. 6f,g depicts that of in sealed-pore case. At each angle of incidence, the incident energy 

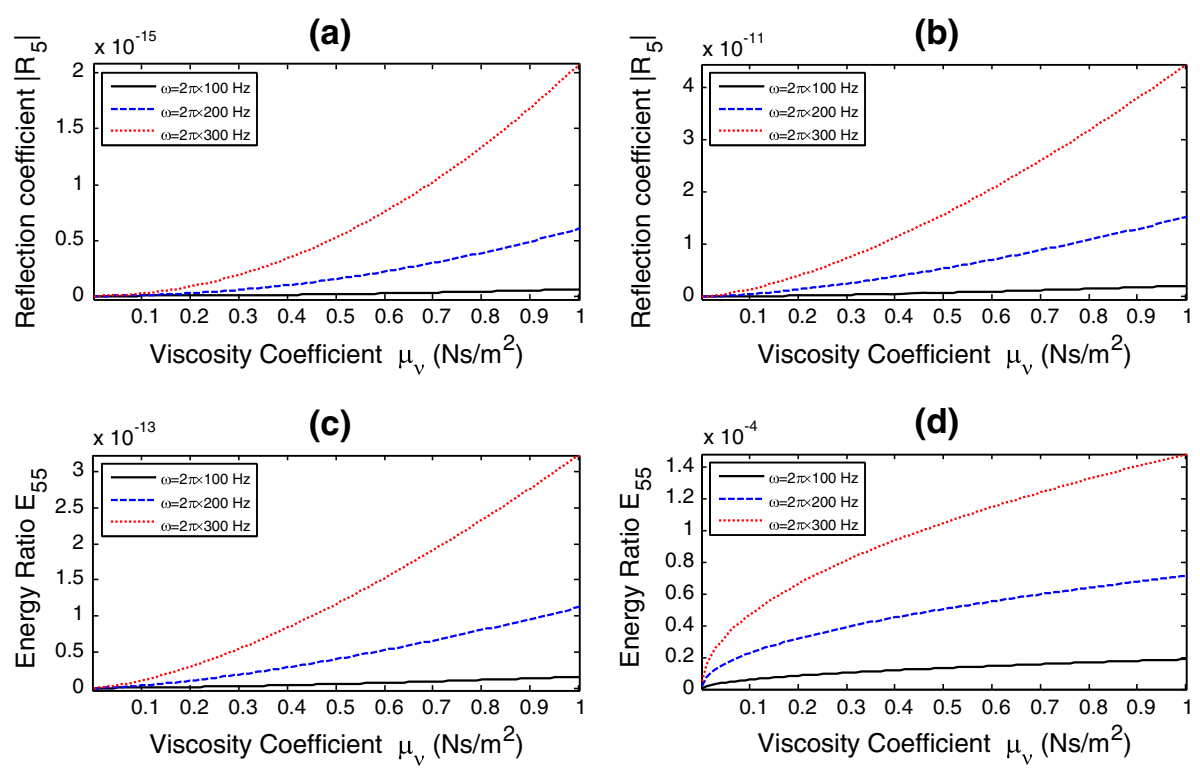

Fig. 7 Variation of $\left|R_{5}\right|$ and $E_{55}$ with viscosity coefficient $\mu_{v}$ : a, b reflection coefficients; $\mathbf{c}$, d energy ratios (at $\gamma_{0}=\theta_{0}=45^{\circ}$ ); $\mathbf{a}, \mathbf{c}$ for open-pore boundary; $\mathbf{b}, \mathbf{d}$ for sealed-pore boundary case

is found to be equal to the sum of energies carried along with various reflected waves and the interacting energies. In both the cases of boundary surfaces, at normal incidence, since only the reflected dilatational wave dominates, therefore the interactional energy between distinct pair of waves is zero. The interaction energies increase as the angle of incidence increases and attain their maximum values as the angle of incidence approaches to grazing incidence. Interactional energies are also immune to the opening/closing of the pores. During numerical computations, again it is found that the reflection coefficients corresponding to reflected shear waves are influenced by the viscosity coefficients of the fluid. A small increase in the value of $\mu_{v}$ leads to significant change in the magnitudes of reflection coefficient of the shear waves. The reflected $S_{2}$-wave is found to be highly influenced, in particular. This can be readily seen through Fig. 7a-d.

In our paper cited above, we have shown that the various results of ET (Eringen's Theory) reduces into TCT (Tuncay and Corapcioglu Theory) when the quantities $\xi^{f g}, \lambda_{v}$ and $\mu_{v}$ are set to zero (almost) numerically in ET. The same observations are made in corrected programme too and the results have been re-displayed through Figs. 8, 9 and 10. Once again it has been seen that in TCT, the phase speed $c_{\beta 2}$ of the second shear wave is very very small and the corresponding attenuation $\left|\mathbf{A}_{\beta 2}\right|$ is very very high, showing that this shear wave cannot be noticed (even in the limiting case) under TCT. Also, reflection coefficient $\left|R_{5}\right|$ vanishes in TCT for both open-pore as well as for sealed-pore boundaries. This shows that one of the reflected shear waves in TCT does not appear, as was expected beforehand. Figure 11 depicts the effect of viscosity coefficient on phase speed and attenuation coefficient of the second shear wave in the considered two fluids (water and crude oil) with different values of viscosity coefficients $\left(\mu_{v}=8.88 \times 10^{-4} \mathrm{Ns} / \mathrm{m}^{2}\right.$ and $\mu_{v}=1000 \times 10^{-3} \mathrm{Ns} / \mathrm{m}^{2}$ approximately). We see that the phase speed (attenuation coefficient) of the second shear wave in the presence of oil is very high (low) than that of in the presence of water in the porous matrix.

The inconvenience caused is highly regretted. 


\section{(a)}

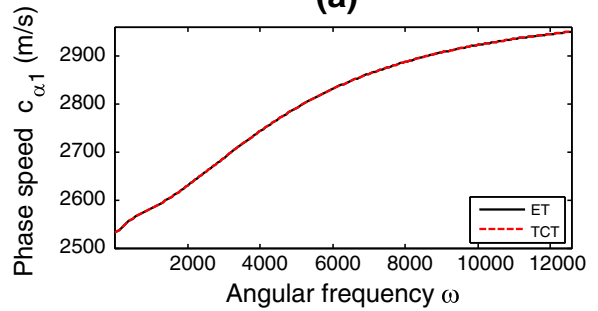

(c)

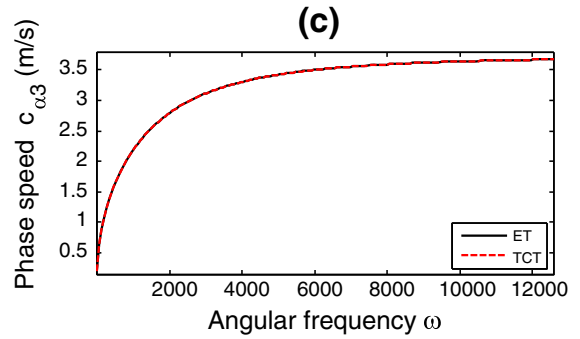

(e)

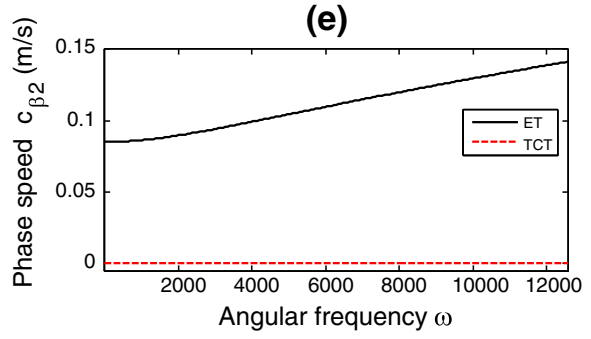

(b)

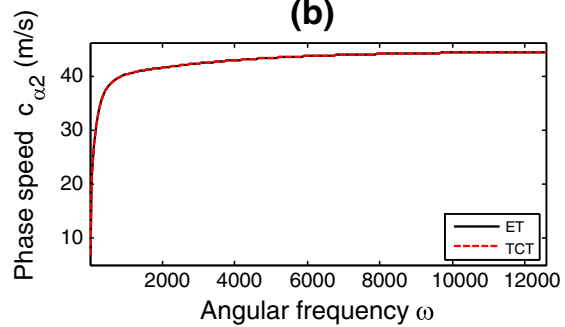

(d)

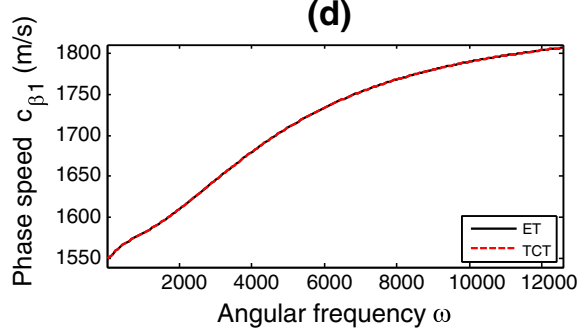

Fig. 8 Phase speeds - comparison between Eringen's theory and Tuncay and Corapcioglu theory (at $\gamma_{0}=$ $\left.45^{\circ}\right)$ 
(a)

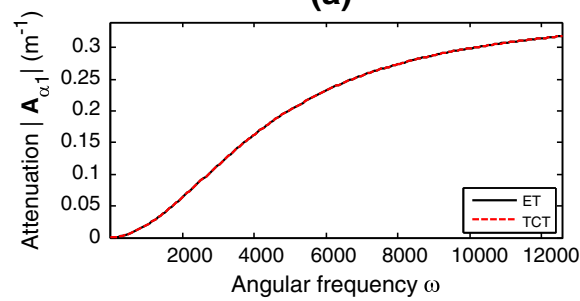

(c)

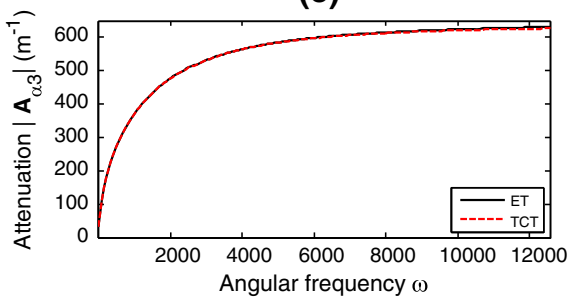

(e)

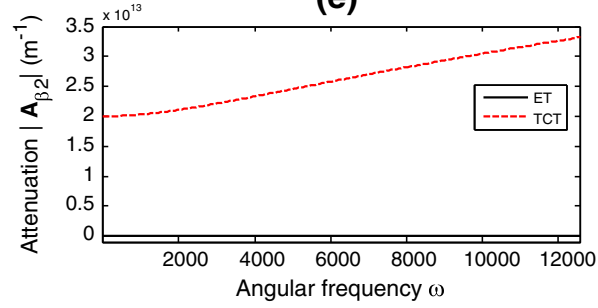

(b)

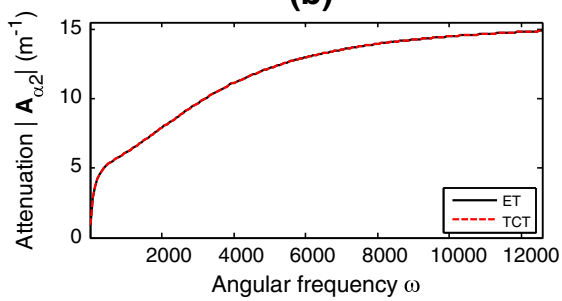

(d)

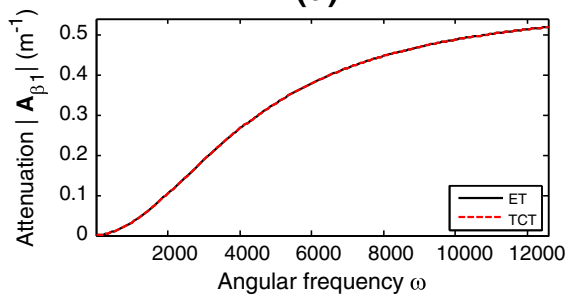

Fig. 9 Attenuation coefficients - comparison between Eringen's theory and Tuncay and Corapcioglu theory $\left(\right.$ at $\left.\gamma_{0}=45^{\circ}\right)$
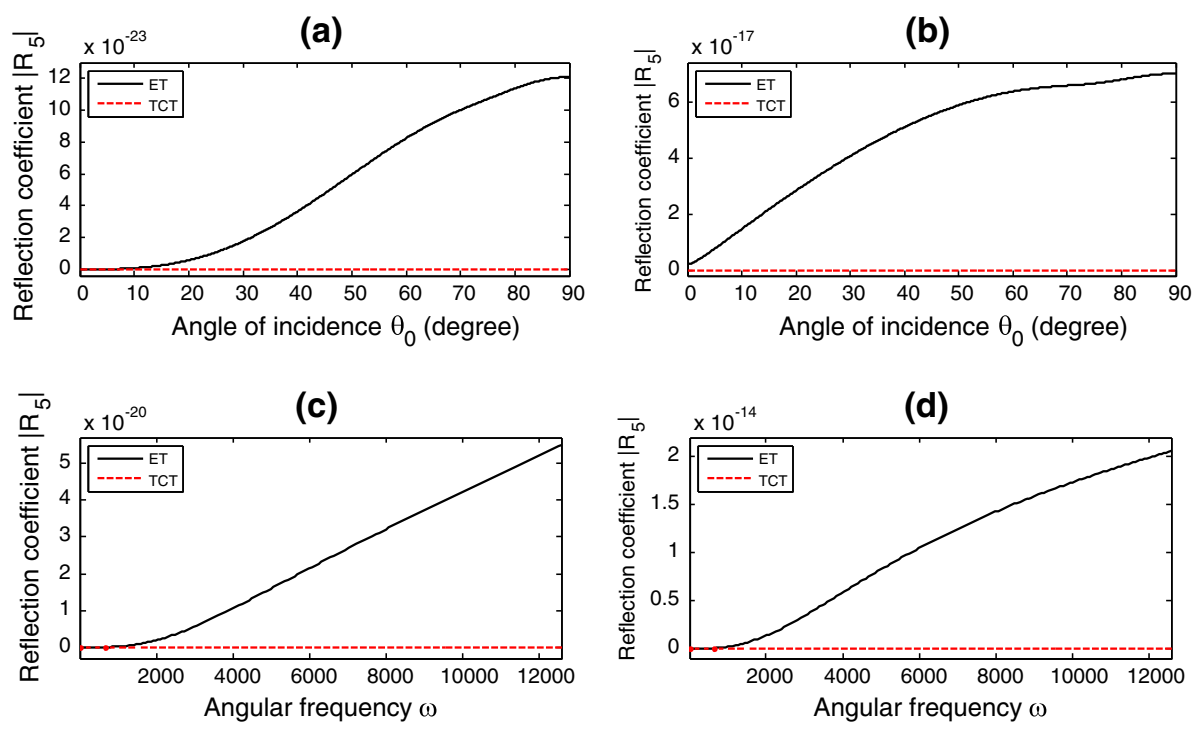

Fig. 10 Reflection coefficients ( $\mathrm{SV}_{2}$-wave) — comparison between Eringen's theory and Tuncay and Corapcioglu theory (limiting case): $\mathbf{a}, \mathbf{b}$ with incidence angle (at $\omega=2 \pi \times 50 \mathrm{~Hz}$ and $\gamma_{0}=45^{\circ}$ ); $\mathbf{c}, \mathbf{d}$ with frequency (at $\theta_{0}=45^{\circ}$ and $\gamma_{0}=45^{\circ}$ ); $\mathbf{a}, \mathbf{c}$ for open-pore boundary and $\mathbf{b}, \mathbf{d}$ for sealed-pore boundary 

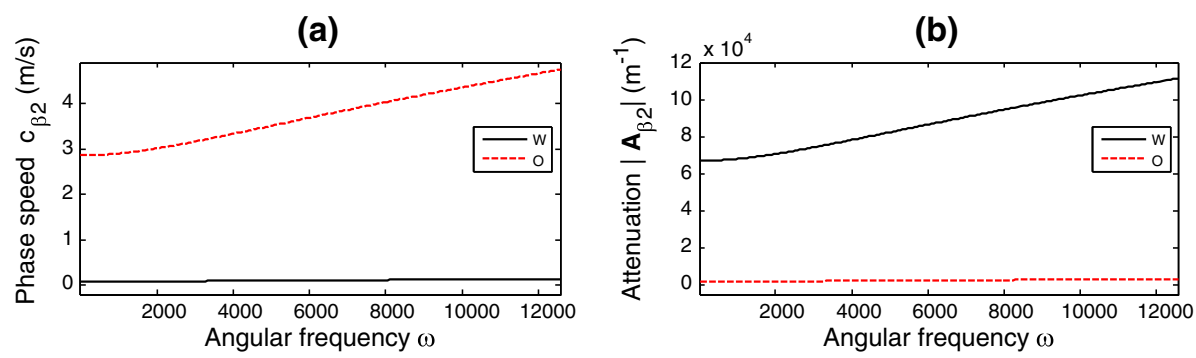

(c)
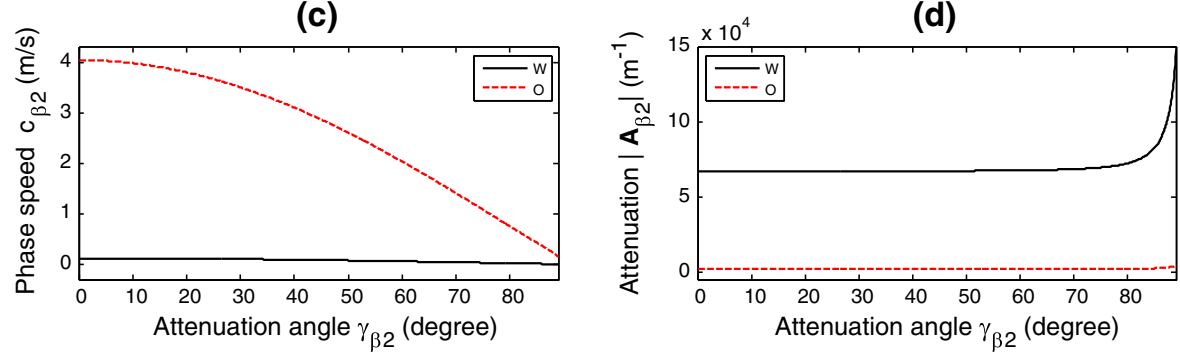

Fig. 11 Phase speeds and attenuations $\left(\mathrm{S}_{2}\right.$-wave $)$ — comparison between water and oil: $\mathbf{a}$, b with frequency parameter $\left(\right.$ at $\left.\gamma_{0}=45^{\circ}\right)$; $\mathbf{c}$, d with attenuation angle $($ at $\omega=2 \pi \times 50 \mathrm{~Hz})$ 